\title{
Estudio de los espacios virtuales como soportes para la violencia de género en la adolescencia
}

\section{Study virtual spaces as supports for gender violence in adolescence}

\author{
María-José Méndez-Lois, Milena Villar-Varela, Felicidad Barreiro-Fernández \\ Universidad de Santiago de Compostela, España
}

\begin{abstract}
Resumen
El objetivo del estudio es visibilizar los espacios virtuales como soportes para la violencia de género en la adolescencia (violencia de género 2.0). Para ello, la USC participa, junto a otras ocho universidades españolas, en un proyecto liderado por la Universidad de Barcelona y financiado por el BBVA, en el que se están recogiendo datos de una muestra de 4500 adolescentes, que cursan $3^{\circ}$ y $4^{\circ}$ de ESO. El instrumento utilizado es un cuestionario electrónico de preguntas en escala tipo lickert. Los resultados servirán para elaborar pautas de intervención y sensibilización ante los comportamientos violentos entre hombres y mujeres jóvenes.

Palabras clave: violencia de género 2.0, tecnologías, diagnóstico educativo, evaluación de necesidades, adolescencia.
\end{abstract}

\begin{abstract}
The objective of the study is to demonstrate the virtual spaces as supports for Gender-based violence in adolescence. For this, the USC participates, along with other Spanish Universities Eight, in a team led by the University of Barcelona. This study is funding by the BBVA in which we are collecting data from a sample of 4,500 teenagers enrolled in 3rd and 4th (high school). The instrument used is an electronic questionnaire in Likerttype scale questions. The results will develop guidelines paragraph intervention and awareness of violent behavior among young men and women

Keywords: 2.0 gender violence, technologies, educational diagnosis, needs assessment, adolescence.
\end{abstract}

La importancia que las redes sociales y las aplicaciones móviles tienen en la adolescencia está siendo objeto de diversos estudios e investigaciones (Estébanez y Vázquez, 2013; Torres, 2013). Las redes sociales han adquirido entre la población adolescente gran importancia lo que las convierte en foco de atención y análisis continuo así como en una preocupación para la sociedad, en general, y para las familias en particular.

Las redes sociales podemos definirlas como comunidades virtuales cuyo objetivo es establecer contacto entre personas. Sin embargo, lo que se inició como un espacio de relación se convierte también en un lugar de socialización y subjetivación (Lásen, 2009). Dentro de las investigaciones realizadas se pone de relieve que las amistades que el o la adolescente tiene en las redes sociales no son tales, sino que las "verdaderas amistades" son un porcentaje pequeño en comparación con las amistades que tienen dentro de sus perfiles. Por tanto podemos hablar de audiencias más que de amistades. Audiencias que muchas veces son difíciles de controlar (Mejías y Rodríguez, 2014) y que en la adolescencia están muy relacionadas con la popularidad, es decir, aquellas personas que son más populares tienen mayores amistades virtuales, mayores audiencias (Estébanez y Vázquez, 2013).

Es evidente que las redes sociales y las aplicaciones móviles se han instaurado, especialmente en la adolescencia, como espacios de relación, espacios de encuentro colectivo que ofrecen nuevas formas de lenguaje, de comunicación... (Bertomeu, 2011). De aquí que se hable actualmente más de TRIC (Tecnologías de la Relación, Información y Comunicación) que de TIC (Gabelas et al., 2012). Otra de las características que definen el uso de las redes sociales y de las aplicaciones móviles es su contribución a lo que Lásen (2009) define como "transformación de la intimidad". Una intimidad que en muchos casos se hace pública a golpe de clic, que provoca un "desnudo" público de la vida afectiva y sentimental en tiempo real (Mejías y Rodríguez, 2014). Este "desnudo de la intimidad" también es facilitado por las propias redes sociales en cuyo perfil se muestra la posibilidad de exponer tu situación sentimental, tu estado afectivo actual...

En esta presentación la corporalidad, la imagen, los modelos de presentación y representación que se muestran, no están exentos de asimetrías de género. Como sostiene Bernárdez: ... construirse una identidad atractiva en la red parece ser una tarea casi tan laboriosa como puede serlo en las interacciones cara a cara (2006: 78). La imagen que muestra la adolescencia en las redes sociales no está exenta de estereotipos de género: mientras que las chicas utilizan estrategias para parecer más atractivas, los chicos lo hacen para parecer más "machos", reforzando así la reproducción de un modelo de feminidad y masculinidad muy anclado en patrones sexistas, en modelos hegemónicos.

Por su parte, las aplicaciones móviles, como por ejemplo WhatsApp, Instagram, Line o Telegram, han constituido una forma de comunicación y relación que excede a las redes sociales. Estar o no estar dentro de esta aplicación móvil u otras, supone quedarse fuera de informaciones que les resultan, a los y las adolescentes, 
de gran interés en su cotidianidad, es decir, quedar con las amistades, comentar un hecho concreto, desconvocar una fiesta... Las redes sociales han creado una necesidad que antes no existía: "como está todo el mundo...", no estar en ellas significa aislarse, perderse cosas (Mejías y Rodríguez, 2014). Si antes la adolescencia quedaba de un día para otro, hoy la inmediatez de aplicaciones móviles como WhatsApp, hace que la flexibilidad esté mucho más presente en torno a cerrar citas, comunicar un hecho, cambiar de planes...

Actualmente para trabajar la violencia de género en las edades adolescentes debemos tener en cuenta las TRIC como espacios cotidianos de interacción, especialmente en las relaciones afectivas (Díaz Aguado, 2013). El estudio realizado por Estébanez y Vázquez (2013) con población adolescente y población joven, concluye que chicas y chicos hacen un uso distinto de las redes sociales, basado en estereotipos y actitudes sexistas que llegan a provocar formas de violencia de género en estas edades. El control ejercido por las parejas, los celos, la prohibición de estar en redes sociales a determinadas amistades, así como el traspaso de contraseñas como muestra de amor y confianza, son algunos ejemplos de maltrato. Es, sin embargo, importante aclarar que las redes sociales o las aplicaciones móviles no producen desigualdad ni violencia de género por sí mismas sino que reproducen las realidades desiguales entre chicas y chicos. Una chica o un chico cuyas relaciones offline, tanto de amistades como amorosas, sean sanas, difícilmente usará de manera machista sus redes sociales y sus aplicaciones móviles. Así, por el contrario la adolescencia que tenga actitudes, pensamientos o valores machistas y que en su cotidianidad desarrolle relaciones de amistad y pareja no saludables, las utilizará como un instrumento más para ejercer ese poder.

La aparición de una vida online desde la que relacionarnos, desde la que iniciar o continuar relaciones tanto de amistad como afectivas, es una realidad en la población más joven. Su presencia en las redes sociales y las aplicaciones móviles muestra que éstas se convierten en un espacio propio y ajeno al mismo tiempo. Propio porque cada cual diseña y usa su vida online de una determinada manera, y ajeno porque cada adolescente interpreta la "identidad digital" de otra persona en función de su escala de valores. El problema estaría, por una parte, cuando la escala de valores de quien presenta un perfil no coincide con la escala de valores de la persona o personas que ven y pueden participar de ese perfil, de su audiencia (Ruiz Repullo, 2014). Pero el problema también lo encontramos cuando dentro de una relación de pareja, una de las dos personas o las dos, quiere hacer del espacio online de la otra persona algo propio. Es decir, cuando aquello que es ajeno se convierte en algo que queremos controlar, que queremos poseer, a través de mecanismos de chantaje, amenazas e insultos. Este es el aspecto que más nos interesaba en relación al uso de las redes sociales y las aplicaciones móviles, su relación con formas de violencia de género, desarrollándose de esta manera un nuevo concepto de violencia: la violencia 2.0. (Donoso-Vázquez, 2014).

\section{El estudio y las personas participantes}

Ante este panorama la Universidad de Barcelona, en colaboración con otras universidades de España, concretamente, la Universidad de Zaragoza, la Universidad de Santiago de Compostela, la Universidad de Valencia, la Universidad de Málaga, la Universidad de Sevilla, la Universidad de Granada (campus Melilla), la Universidad de las Islas Baleares y la Universidad de La Laguna piensa en llevar a cabo un proyecto sobre las violencias de género 2.0, financiado por la Fundación BBVA con una duración de dos años. El equipo de investigación está formado por 26 investigadoras e investigadores vinculados a las universidades citadas. Es de destacar que cada equipo de las nueve universidades participantes tiene una especialización socioeducativa específica, que aporta experiencia e innovación al tipo de análisis que se desarrollará en los diferentes momentos o fases de la investigación.

\section{Método y objetivos}

La hipótesis de partida del proyecto es que hay elementos diferenciadores entre las violencias de género que se dan fuera de línea y las que se dan online. Estos elementos diferenciadores vienen dados tanto por el flujo de las relaciones virtuales como por las dinámicas que se establecen y por el grado de expansión de las agresiones. Las violencias en función del género son aquellas agresiones contra las mujeres por el hecho de ser mujeres y contra aquellas personas que transgreden la heteronormatividad de género (Momoitio, 2014).

El estudio propone en primer lugar, un análisis diagnóstico y en segundo lugar una evaluación de necesidades entorno a las violencias de género 2.0. El diagnóstico versará sobre las percepciones de la juventud respecto a Internet y la violencia 2.0, las experiencias que han tenido en violencia 2.0, y las respuestas que han protagonizado en dichas experiencias. Concretamente, son de gran importancia las experiencias de las y los jóvenes en otras violencias cara a cara, así como su perfil digital.

Esta primera fotografía nos permitirá focalizar mejor la dinamización de un foro participativo donde la juventud podrá expresar, en su propio lenguaje y forma, sus necesidades expresadas respecto a las violencias de género 2.0. Entre las posibles expresiones están las tiras cómicas, los memes, las imágenes gifs, los videos en youtube o facebook, las pintadas, los lipdubs, las causas en change.org, verkami o similares, $y / u$ otras manifestaciones artísticas o narrativas digitales. Es especialmente importante el análisis de estas expresiones de las y los jóvenes para escuchar en su propia voz los elementos intrínsecos de la realidad de las violencias de género 2.0 que sufren y los mecanismos de prevención y actuación ante este fenómeno, desde su mirada.

Ambas aproximaciones deben permitir un diagnóstico en profundidad a cerca de la vulnerabilidad y las necesidades expresadas de las y los jóvenes ante las 
violencias de género 2.0. A partir de los resultados del proyecto se pretende establecer un modelo descriptivo sobre las violencias de género que se dan en los espacios virtuales que aporte nuevos elementos al debate conceptual de las violencias de género en plena sociedad digital y líneas de acción para combatir la violencia de género 2.0. La detección de las necesidades expresadas de las y los jóvenes debe permitir ofrecer pautas educativas de sensibilización, prevención y actuación ante tales violencias. Desde el punto de vista de responsabilidad social de la investigación responde a la llamada de ofrecer indicadores que permitan establecer estrategias de protección a las personas susceptibles de sufrir violencia de género en la red.

Estas finalidades se operativizan con los siguientes cinco objetivos generales y sus objetivos específicos respectivos:

1. Objetivo 1. Analizar las percepciones de la juventud sobre Internet y telefonía móvil ante la violencia 2.0 en diferentes regiones españolas.

Este objetivo tiene los siguientes objetivos específicos:

- Identificar las percepciones de la juventud sobre los riesgos presentes en las redes.

- Analizar la percepción de la juventud sobre la violencia presente en Internet y redes sociales.

2. Objetivo 2. Analizar las experiencias que ha tenido la juventud en cuanto a formas de violencia de género 2.0 en diferentes regiones españolas.

Este objetivo tiene los siguientes objetivos específicos:

- Identificar la incidencia y comportamientos de ciberacoso desde la perspectiva de las víctimas.

- Identificar el ciberacoso desde la perspectiva de los agresores.

- Analizar las respuestas de adolescentes y jóvenes en sus experiencias en violencias de género 2.0.

3. Objetivo 3. Identificar perfiles de vulnerabilidad ante las violencias de género 2.0 en diferentes regiones españolas.

Este objetivo tiene los siguientes objetivos específicos:

- Identificar las variables que pueden favorecer esta vulnerabilidad.

- Analizar si existe relación entre perfiles de violencia en general y perfiles de violencias de género 2.0.

- Analizar la relación entre el perfil tecnológico y la vulnerabilidad.

4. Objetivo 4. Analizar la voz de las y los jóvenes ante las violencias de género 2.0 en las diversas manifestaciones producidas, detectando algunas necesidades expresadas relativas a las violencias de género 2.0 por parte de las y los jóvenes en un entorno virtual, e identificando los elementos intrínsecos del fenómeno.

Este objetivo tiene los siguientes objetivos específicos:

- Desarrollar un entorno virtual donde las y los jóvenes puedan expresar sus opiniones y vivencias ante las violencias de género 2.0, dinamizado desde el proyecto.

- Facilitar diversos canales, recursos y lenguajes de expresión en el entorno virtual (memes, tiras cómicas, gifs, videos, lipdubs, causas, etc) de manera que los y las jóvenes puedan ser prosumidores (productores y usuarios) de la plataforma de violencias de género 2.0.

- Analizar las narrativas digitales y otras expresiones de las y los jóvenes sobre violencias de género 2.0.

5. Objetivo 5. Elaborar pautas educativas de sensibilización, prevención y actuación ante las violencias de género 2.0, para jóvenes, familias, agentes educativos $\mathrm{y}$ organizaciones gubernamentales.

Este objetivo tiene los siguientes objetivos específicos:

- Difundir los resultados obtenidos para sensibilizar a la población sobre la problemática.

- Elaborar pautas preventivas específicas de violencias de género 2.0.

- Elaborar pautas de intervención ante casos de violencia de género 2.0 .

\section{Fases del estudio}

La investigación consta de cuatro grandes fases:

En la primera tiene lugar la elaboración y revisión del cuestionario online, y su aplicación al alumnado de tercero y cuarto de la Educación Secundaria Obligatoria, previa autorización del centro. Además, una vez cumplimentados los cuestionarios, se procederá al análisis de datos y a la obtención de resultados.

En la segunda fase se lleva a cabo un proceso de sensibilización del alumnado teniendo en cuenta los resultados obtenidos en la fase anterior. Para ello, las personas que conforman cada uno de los nueve equipos de trabajo diseñarán diversos talleres que serán llevados a cabo en los centros en los que se haya recogido la información.

La tercera fase hace referencia a la dinamización de los institutos, en ella el alumnado dará a conocer su voz a través de las propuestas creativas y los concursos entre todos los centros participantes.

La última fase se centra en las conclusiones y el desarrollo de pauta educativas. Los resultados obtenidos serán útiles para ampliar el conocimiento de la comunidad científica y difundirlo a través de congresos, artículos de revistas o plataformas web, lo que conllevará a una continuación futura en la investigación del problema y en la creación de formación online para subsanar las necesidades formativas de la población. Pero no se trata solo de una difusión entre personal experto, sino que se pretende que los resultados del estudio salgan a la luz en los medios de comunicación generales, para que la sociedad detecte estas anomalías conductuales y luche por un cambio a gran escala.

\section{Conclusiones}

Actualmente la investigación está comenzando, nos encontramos en la primera fase, concretamente en el momento de aplicación de los cuestionarios al alumnado 
de $3^{\circ}$ y $4^{\circ}$ de la ESO para la recogida de datos. En cuanto a la muestra, se espera recoger información de un total de 4500 alumnas y alumnos pertenecientes a las nueve regiones que participan en el estudio.

En la Universidad de Santiago de Compostela, el equipo de trabajo está constituido por cuatro investigadoras especialistas en educación, con adecuada formación en perspectiva de género y con una clara vocación feminista. Nuestro aporte al proyecto se centra principalmente en colaborar desde nuestra experiencia en investigación cuantitativa y cualitativa, orientación educativa y estudios de género que esperamos sirva para enriquecer no sólo los instrumentos de investigación que se utilizan sino también las propuestas innovadoras que se desarrollen tanto preventivas con la población juvenil ante el uso de las tecnologías de la comunicación, como de intervención con mujeres jóvenes que sufran o hayan sufrido violencia 2.0.

En cuanto a los resultados teniendo en cuenta los datos de otros estudios precedentes (Ruiz Repullo, 2014) esperamos poder ofrecer información sobre la presencia que tienen las aplicaciones tecnológicas en la muestra de adolescentes consultados y si las redes sociales y las aplicaciones móviles se configuran como espacios para ejercer violencia de género.

El uso de indicadores como el control, el chantaje, las prohibiciones o las amenazas, incluidos en los ítems del cuestionario elaborado nos permitirá obtener datos sobre su presencia y su frecuencia entre los y las adolescentes a los que preguntamos. Además es nuestro propósito saber si hay diferencias en las percepciones de las personas consultadas cuando los datos que ofrecen se personalizan y los responden desde distintas posiciones en el uso del poder ante las situaciones que contestan (agresor/a, víctima y/o observador/a).

Debemos ser conscientes, así mismo, de que si la desigualdad y la violencia son palpables en el espacio virtual esto nos debe hacer estar alerta sobre la presencia de la misma en la vida real. El desarrollo de la vida online y offline dentro de las relaciones de pareja adolescentes es complementaria y creemos que es fácil que, lo que ocurre en una se traslade a la otra y viceversa. Será muy necesario trabajar con los y las adolescentes que crean que los espacios virtuales y/o la tecnología son los que provocan problemas en sus relaciones sin pararse a pensar que esos problemas no son producidos por las aplicaciones sino por sus formas de relacionarse afectivamente.

\section{Referencias}

Bernárdez, Asunción (2006). A la búsqueda de una 'habitación propia': comportamiento de género en el uso de Internet y los chat en la adolescencia. Revista de Estudios de Juventud. Adolescencia $y$ comportamiento de género, núm. 73, Junio, 69-82.

Bertomeu, Angustias (2011). Nativos digitales: una nueva generación que persiste en los sesgos de género. Revista de Estudios de Juventud. Adolescentes Digitales, núm. 92, Marzo, 187-202.

Díaz Aguado, Ma José (Dir.) (2013). Evolución de la adolescencia española sobre la igualdad y la prevención de la violencia de género. Madrid: Ministerio de Sanidad, Política Social e Igualdad. Centro de Publicaciones.

Donoso-Vázquez, Trinidad (Coord.) (2014). Violencias de género 2.0. Barcelona: Kit-book.

Estébanez, Ianire y Vázquez, Norma (2013). La desigualdad de género y el sexismo en las redes sociales. Bilbao: Servicio Central de Publicaciones del Gobierno Vasco.

Gabelas, Juan Antonio; Lazo, Carmen Marta y Aranda, Daniel (2012). Por qué las TRIC y no las TIC [En línea]. Revista de los Estudios de las Ciencias de la Información y la Comunicación, núm. 9, Marzo. Barcelona: UniversitatOberta de Catalunya. Recuperado de http://www.uoc.edu/divulgacio/comein/es/numero09/a rticles/Article-Dani-Aranda.html

Lasén, Amparo (2009). Tecnologías afectivas: de cómo los teléfonos móviles participan en la constitución de subjetividades e identidades. En Gabriel Gatti, Iñaki Martínez de Albéniz y Benjamín Tejerina (Eds.). Tecnología, cultura experta e identidad en la sociedad del conocimiento, 215-248. Bilbao: Universidad del País Vasco.

Mejías, Ignacio y Rodríguez, Elena (2014). Jóvenes y comunicación. La impronta de lo virtual. Madrid: Centro Reina Sofía para Adolescentes y Juventud y Fundación de Ayuda a la Drogadicción (FAD).

Momoitio, Andrea (2014). Internet al servicio de la violencia contra las mujeres. En Trinidad DonosoVázquez (Coord). Violencias de género 2.0. Barcelona: Kit-book, 13-28.

Ruiz Repullo, Carmen (2014). La construcción social de las relaciones amorosas y sexuales en la adolescencia. Graduando violencias cotidianas. Jaén: Diputación provincial de Jaén.

Torres, Cristóbal (Dir.) (2013). El ciberacoso como forma de ejercer la violencia de género en la juventud: Un riesgo en la sociedad de la información $y$ del conocimiento.Madrid: Ministerio de Sanidad, Servicios Sociales eIgualdad-Centro de Publicaciones. 